\title{
Punctual professor using passé procedures
}

At exactly 10:00 a.m. every morning Dr. Conrad Vogelman entered the animal facility at Great Eastern University. He carefully removed one cage of rats from the animal holding room, brought it into the procedure room, and began his studies. This is the pattern he had followed for nearly 20 years-never early, never late, always at 10:00 a.m. Vogelman was a neuroscientist who methodically studied drugs that cause euphoria, trying to discern the mechanism by which the drugs induce dependence. In his rat model he would inject the animals with varying dosages of drugs and would then record the brain activity from previously implanted electrodes.

This was Vogelman's daily routine. At nearly 85 years of age, he had retired some 15 years earlier as an emeritus professor. Although he was no longer in the mainstream of research, his gentle demeanor and generosity toward students were highly prized by Great Eastern. In turn, the Department of Neuroscience had supported his research for many years, because Vogelman's work was not of sufficient quality to attract external funding. His colleagues knew that most of the techniques he used were passé, but he was so well liked that nobody could bear to tell him to stop, even though none of his work had been published since before his retirement.

Of course, the Great Eastern IACUC routinely reviewed and approved Vogelman's research. His animals received the best of care, he performed the surgical implants flawlessly and humanely, and followed the protocol meticulously; overall, Vogelman was an exemplary investigator. The only problem (if, indeed, there was a problem) was with the research itself. For 20 years there had been IACUC review of Vogelman's work. For 20 years he had used animals. For 20 years nothing emanated that was of publishable quality. What was the value of this research? The IACUC had, on more than one occasion, discussed the merit of Vogelman's science, and invariably concluded that because the mechanism of drug dependence was not fully understood, his animal use procedures met regulatory standards and, despite the absence of funding and publications, he was asking a valid scientific question. So, Vogelman's work continued every morning, every day, at 10:00 a.m.

The question is, should the IACUC have said that there was little or no scientific merit in these studies and it was time for Vogelman's research to be retired? Furthermore, if the Committee came to such a conclusion, could it refuse to approve his research?

\section{RESPONSE}

\section{Answer unclear}

\section{Alan L. Rothman, MD}

We must consider here two specific questions. The first is whether or not the IACUC should have said there was little or no scientific merit to the proposed research, and the second is whether or not, after coming to such a conclusion, the IACUC could refuse to approve the research. The second question is straightforward. The IACUC has no obligation to approve any research. The first question is less straightforward. At the time of protocol review the IACUC should consider whether the proposed research is relevant to health or the advancement of general knowledge and whether the approach used minimizes both the number of animals used and the pain and distress to the animals to the extent possible consistent with the scientific objectives. This implies that there are scientific objectives of some merit for the advancement of knowledge. If this work is truly without merit, then it should not take place, and the IACUC should reject the proposal.

We can only speculate on the content of Vogelman's protocol. We know that the protocol involves survival surgery on rats, injection of drugs, and monitoring. We also know that the animal care and technical skills used were "exemplary." Thus, assuming that there is some merit in the research proposed, animal welfare seems not to be an area of concern.

The information provided about the merit of the proposed work is conflicting. The research has neither been published nor received external funding for an extended time. On the other hand, the IACUC concluded that the research was asking "a valid scientific question." Are these two assessments contradictory? If not, then which should prevail? $\mathrm{NIH}$ review panels sometimes follow popular trends rather than evaluating purely on the basis of scientific merit, a common lament of scientists considered outside the "mainstream." The same process influences decisions about the publication of manuscripts in scientific journals. If Vogelman follows good scientific procedure, he should be documenting his observations carefully; it is possible that these notes would be of use to advance scientific knowledge in the future. If it is valid for an IACUC to question the validity of proposed research that has received external approval and funding, then the converse should also be possible. The proposed research can (and should) be held to the standard of scientific merit even without confirmation by an external review. Perhaps the willingness of his department to support this research reflects more than a sense of debt.

One additional point is worthy of discussion. His colleagues apparently felt that his techniques were outmoded. This raises a question whether the experiments proposed meet the relevant standards. Techniques can become unpopular but still 